\title{
Research on Maintenance Free Operating Period
}

\author{
CUI Yu Lian \\ Mechanical Engineering Department of Academy of Armored Forces Engineering, Beijing, 100072 \\ cuicuilili@hotmail.com
}

\begin{abstract}
Keywords: MFOP; basic concept; basic idea; advantages and benefits; disadvantages and risks Abstract: Since MTBF has several drawbacks, maintenance free operating period (MFOP) is proposed for it is better, cheaper and faster. The paper analyses the basic concept of MFOP, the basic ideas to achieve MFOP, the advantages and benefits that MFOP brings about, the disadvantages and risks that MFOP faces.
\end{abstract}

\section{Introduction}

As we all know, the classic definition of reliability is "The ability of an item to perform a required function under given conditions for a given time interval," or "Number of failures permitted under given conditions for a given time interval." Under this definition, Mean Time Between Failures (MTBF) or failure rate is commonly used when specifying reliability requirements in the past. It virtually considers that random failures are inevitable and acceptable and allows the existence of a certain percentage of failures which results in incomplete research of the failures, for example, failures due to design fault can not be traced to the ultimate sources as long as they are in the allowable range, and failures due to manufacturing process would not be investigated as long as its number is lower than the number permitted. Therefore, people deviate their attentions away from investigating the failure mechanism completely and tolerate the existence of some unexplained failures provided that such failure does not exceed the permitted numbers and characteristic boundaries. This not only causes some errors in the analysis and calculation, but also causes the customers at risk of failures at any time which led to the occurrence of numbers of unscheduled maintenance work. Unscheduled maintenance has become one of the main problems the equipment currently faces, which reduces the availability of the equipment, so decreases the forces combat capability. And due to time constraints, the cost of unscheduled maintenance is usually much higher than that of planned maintenance. Numerous of unplanned maintenance has been a huge challenge to the equipment affordability. Because the equipment affordability factors include not only the acquisition cost, but also the maintenance and support cost.

In addition, at present, the design reliability level is approaching a limit. If the traditional design methods are still to be used, it becomes very difficult to increase the reliability level even a little bit.

Therefore, there is a need to improve the metric of reliability and maintainability. The measure should be of interest to users and can be used to optimize the system design. In order to reach his goal, Maintenance Free Operating Periods (MFOP) were first proposed by the MOD. MFOP is not a purely technical indicator or metric, it is a concept that can reflect the customer's final demand.

\section{Basic Concept of MFOP}

MFOP is defined as a period of operation during which the equipment must be able to carry out all its assigned missions without any maintenance action and without the operator being restricted in any way because of system faults or limitations [1]. It should be noted that an MFOP is not defined to contain absolutely no maintenance, rather it is considered that faults and minor planned, contractually agreed maintenance are permissible, such as refueling, rearming and repairing important safety related features[2]. The prime objective of maintenance is to ensure that a product can perform its intended functions. Every MFOP is followed by a maintenance recovery period (MRP), i.e. the downtime during 
which the equipment is recovered to such a level that the next MFOP can be achieved successfully. Under the philosophy of MFOP, unscheduled corrective maintenance could be avoided by employing fault-tolerant, redundant, and reconfigurable systems. Only scheduled preventive maintenance should be carried out in previously arranged MRP.

The philosophy of MFOP assumes that success is attainable and that this probability can be accurately applied from when the equipment enters service. In practical terms, in order to measure the probability that a mission can be successfully completed without maintenance during MFOP, the concept of MFOP survivability (MFOPS) is used. MFOPS is then defined as the probability that the item will survive for the duration of the MFOP. In other words, it is the probability of not having any unscheduled maintenance for a period of tmf life units given the current age of the item [3]. It is also the probability that the item maintains its functionality at least for a period of tmf life units without the need for corrective maintenance due to failure of a component of the system which results in an overall critical failure of the system.

Take a one-unit system for instance, and suppose that the failure mode of the system can be modeled by a two-parameter Weibull distribution. According to the definition of MFOPS, if the reliability requirement is MFOP of tmf life units, the probability of surviving tmf units of time given that the system has survived $t$ units of the time is given by

$$
M F O R(S)=\frac{R\left(t+t_{m f}\right)}{R(t)}=\exp \left[\frac{t^{\beta}-\left(t+t_{m f}\right)^{\beta}}{\eta^{\beta}}\right]
$$

where $\eta$ is the scale parameter and $\beta$ is the shape parameter of the Weibull distribution. $R(t)$ refers to the reliability of the system, which is given by

$$
R(t)=\exp \left[-\left(\frac{t}{\eta}\right)^{\beta}\right]
$$

MFOPS prediction model can be used as a reliability measure to help to decide maintenance strategy.

The concept of maintenance free operating period (MFOP) is not new, it is essentially same as the warranty period. What is new is that the operators are considering extending this concept throughout the life of the system. In MFOP manufacturer will be asked to guarantee that no unscheduled maintenance activities will be needed. In order to derive the MFOP with the required level of confidence, some life tracking and reliability improving in product need to be ensured.

\section{Basic Ideas of Achieving MFOP}

As shown in Figure 1, MFOP can be regarded as a safe operation time window at a certain confidence level. In order to achieve or increase the time span of the window, in the operating period, it should be possible to reduce or avoid failures or accommodate the failures after they occurred. To achieve such requirement, it can be considered from the following three aspects.

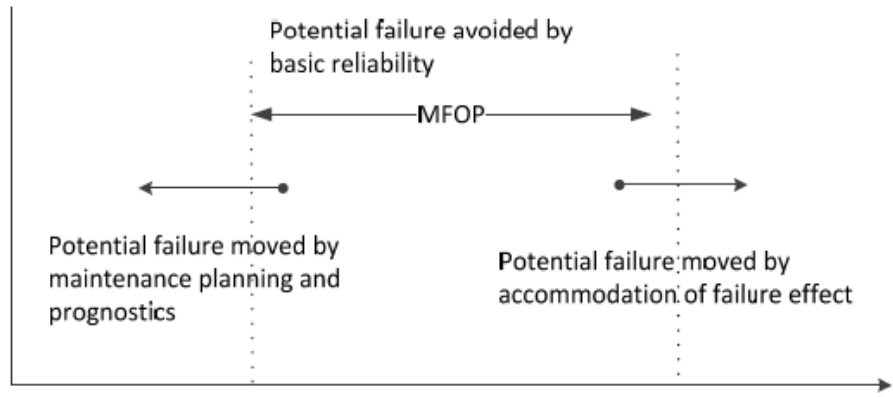

Fig. 1 Basic ideas of achieving MFOP 
Avoid failures. This means improving the basic reliability of system and components, reducing the probability of failures, minimizing the failures of the operating period as far as possible.

Forecast failures. This means applying prognostics techniques and preventive maintenance, replacing components that may go wrong in the next MFOP in advance and eliminating hidden faults, thereby avoiding malfunction during use;

Accommodate failures. This means integrating redundancy, diagnostics and reconfiguration techniques to identify and accommodate failures resulted in the operating periods, and deferring maintenance activities until the end of this MFOP.

So Areas Critical to the MFOP include: reliability design, condition monitoring, prognostics and diagnostics, redundant systems, reconfigurable systems, recovery blocks and self-healing, and reversionary modes, etc.

\section{Advantages and Benefits of MFOP}

The main advantages of using MFOP include the predictability of maintenance periods which allows for overheads such as manpower and facilities to be used less often and with more planning, with little probability of emergency or reactive maintenance [4]. And products can be available throughout a mission or series of missions, saving on costs or allowing for greater flexibility in mission planning.

The benefits of using MFOP is cost related as it is making maintenance to be more efficient, by employing more powerful diagnostic tools, or planning repairs and spares better. First, operational effectiveness would be greatly enhanced because a system would only require specific maintenance levels at a pre-determined period. In addition, logistics support and repair costs could be minimized with maintenance downtime preprogrammed around operational commitments [5]. Actually it can maximize benefits and savings from COTS technology by instituting a system support change technology by instituting a system support change management approach, i.e. MFOP. Other potential benefits include the following: Reliability of contracts would improve because the MFOP is a simpler concept than MTBF. Random component failures would be reduced. True causes of failure would be identified because of the physics approach, rather than statistical analysis involving MTBF. There would be a greater understanding of failure mechanisms and subsequent development of necessary design tools. And using the principle of a failure free period rather than failures randomly occurring would alter the basis of logistics planning. Compared with using reliability predictions based on constant failure models, more realistic spares provisioning should be possible, and expensive, inconvenient unscheduled maintenance should be minimized.

\section{Disadvantages and Potential Risks of MFOP}

While there is much that could be gained from the use of MFOP, there are also potential downsides. For example, MFOP is based on a hierarchy whereby the module of product with the smallest MFOP defines the MFOP of the product. This allows for the most unreliable module of product to be improved, after which the product with the next lowest MFOP is improved, and so on. Their eventual quality was poor, as they did not focus on the overall reliability of the product but its constituents. This approach therefore has a historical basis to be considered as weak. High level of improvements and technologies which will have to be developed to allow the MFOP concept to be successful is very ambitious and will take much time, money and effort to carry out.

Alternatively, potential risks were also acknowledged. Immigrating from MTBF to the MFOP, inspection or refurbishment requirements for some parts may be increased while other components may be scrapped before the end of their previously used life. As a result, each component, line replaceable unit (LRU), and system would require some design analysis to establish its optimum MFOP and associated cost. Under this scenario, modeling to determine potential manpower savings was difficult. 
Other risks include: Increased acquisition costs from a more rigorous design process. Extensive analysis, conducted by skilled technicians, would have to be done because a large number of individual LRUs, subsystems, and system MFOPs into an overall system MFOP would have to be aggregated and understood completely. An integrated knowledge of engineering process design, an appreciation of practical in-use problems, and an understanding of statistics would be required to gain a deeper understanding of the MFOP concept.

\section{Summary}

Since MFOP has the potential that can significantly improve the operational capability and reliability of the product applying it and therefore provides a way of meeting the customer's needs better, many reliability researchers have paid attention to it. Though the proposed time of maintenance free operating period concept is not long, it will come to be understood. For stringent project reliability requirements, it is likely that MFOP will replace MTBF. Compared to MTBF, MFOP can better satisfy customers' requirement of completing assigned mission and meanwhile reducing Life Cycle Cost. And the prominent advantage of MFOP is that it reflects or tracks the entire life status of the system, and encourages the suppliers to analyze every failure mechanism of the equipment they supplied, so the design and reliability can be improved. But it should also be recognized that although MFOP has many advantages and brings benefits, it also faces challenges when considering the disadvantages and risks.

\section{References}

[1] Hockley C J, Appleton D P. Setting the requirements for the Royal Air Force's next Generation aircraft, Reliability and maintainability symposium, 1997: 44-48.

[2] J Long, R A Shenoi, W Jiang. A reliability-centered maintenance strategy based on maintenance-free operating period philosophy and total life time operating cost analysis. Proc. IMechE Vol. 223 Part G: J. Aerospace Engineering, 2009, pp711-719.

[3] Dinesh Kumar, U., Knezevic, J., and Crocker, J., Maintenance free operating period - an alternative measure to MTBF and failure rate for specifying reliability? Reliability Engineering and System Safety, Vol. 64, 1999, pp. 127-131.

[4] Relf M N. Maintenance-free operating periods-the designer's challenge. Quality and reliability engineering international 1999, Vol. 15, pp.111-116.

[5] Xiaohua Wen,et al. An Aircraft Maintenance Free Operating Period Allocation Approach.IEEE, 2014

[6] Zhanyong Ren,Yueqin Wu. Modeling the Performance of Aircraft Utilizing Maintenance Free Operating Periods. Procedia Engineering 99, 2015, pp 214 - 218 